\title{
INGESTA DE ALIMENTOS DE ORIGEN ANIMAL VERSUS ORIGEN VEGETAL Y RIESGO CARDIOVASCULAR
}

\section{INTAKE OF ANIMAL VERSUS VEGETABLE FOOD AND CARDIOVASCULAR RISK}

\author{
Ángela Pino L., Gustavo Cediel G., Sandra Hirsch B. \\ Instituto de Nutrición y Tecnología de los Alimentos (INTA), Universidad de Chile. Santiago, Chile.
}

\begin{abstract}
Cardiovascular mortality has been associated with changes in lifestyle and food habits. The occidental diet has been characterized by high intake of animal fat and meat, and it is associated whit increased risk of type 2 diabetes and high blood pressure, an increase in levels of markers of endothelial dysfunction, and presence of prothrombotic risk factors. We search the Pubmed database and other review references. Inclusion criteria were: papers published after 1999, randomized controled clinical studies, case-control studies, systematic reviews and methanalyses. Vegetarians groups show less cardiovascular morbidity and mortality than non-vegetarians. The evidence indicated that a decreasing intake of red meat, saturate fat, trans fat, and mainly processed meat, and an increase in the intake of whole grain cereals, fruits, vegetables and fish, are associated with a better overall cardiovascular health and survival.
\end{abstract}

Key words: Animal foods; vegetable foods; high blood pressure; DM2; cardiovascular mortality.

Este trabajo fue recibido el 8 de Abril de 2008 y aceptado para ser publicado el 10 de Julio de 2009.

\section{INTRODUCCIÓN}

Desde hace aproximadamente dos millones de años el humano ha aumentado el consumo de carne, durante este tiempo la selección natural ha permitido la adaptación de nuestra fisiología a una dieta rica en proteínas, baja en grasas totales, saturadas y relativamente rica en ácidos grasos poliinsaturados (PUFA) (1). Sin embargo es contradictorio pensar que si la evolución ha permitido la selección de seres humanos preparados para el consumo de alto contenido de alimentos de origen animal, la evidencia muestre que la morbi-mortalidad total, principalmente por cardiopatía coronaria sea significativamente menor en vegetarianos comparado con los no vegetarianos (2). El objetivo de esta revisión es conocer los estudios publicados de mortalidad y riesgo cardiovascular asociado a la ingesta de alimentos de origen animal versus los de origen vegetal.

\section{MÉTODOS}

La búsqueda se realizó en la biblioteca online Pubmed y en referencias de otras revisiones. Los descriptores fueron: "animal food", "vegetable food", "cardiovascular" y "risk". Se utilizaron los integradores AND y OR.

Los criterios de inclusión fueron: artículos de 1999 en adelante, ensayos clínicos aleatorizados y controlados, caso y control, revisiones sistemáticas y meta análisis.

\section{RESULTADOS}

Los resultados se describieron a partir de la asociación entre el consumo de alimentos de origen vegetal versus origen animal y riesgo cardiovascular abordando las principales causas: la diabetes mellitus tipo 2 y la hipertensión, posteriormente los factores en investigación intensiva por su rol en el estudio del prevención de riesgo cardiovascular: La composición de los ácidos grasos de la dieta, los factores de riesgo trombótico, y los marcadores de inflamación y disfunción endotelial. Por último se describieron los estudios relacionados con mortalidad cardiovascular. 
Diabetes mellitus tipo 2 (DM 2)

La adopción del estilo de vida occidental está fuertemente asociado con diabetes tipo 2 (3), debido a la falta de actividad física y la presencia de hábitos dietarios inadecuados. Estudios en animales sugieren que el tipo de grasa en la dieta puede afectar la insulino sensibilidad por cambio en la composición de ácidos grasos en las membranas lipídicas. La proporción de grasa insaturada en las membranas del músculo esquelético se asocia con insulino sensibilidad en humanos (4) Diversos estudios de hiperinsulinemia e hiperglicemia muestran un efecto negativo de las grasas saturadas y un efecto beneficioso de grasas poliinsaturados $(5,6)$. Pocos estudios han examinado el posible rol de los ácidos grasos w3 o ácidos grasos trans, en el desarrollo de diabetes tipo 2 (6-8).

En un estudio de doce años de seguimiento con una muestra de 42.504 mujeres profesionales de la salud entre 40 y 75 años, se evidenció la asociación entre el consumo de grasas totales, saturadas y carnes procesadas con alto riesgo de diabetes tipo 2, independiente del índice de masa corporal (IMC) (5). El consumo de carne también se asoció con alto riesgo de diabetes en el estudio de los adventistas del séptimo día (9). y además, en el Nurses' Health Study el consumo de carnes procesadas se asoció positivamente con riesgo de diabetes tipo 2, luego de ajustar por IMC, ingesta de alcohol y consumo energético.

Los nitritos se usan comúnmente en la preservación de carnes, siendo la mayor fuente en la dieta (10). Las nitrosaminas pueden ingerirse en los alimentos o formarse en el estómago por interacción de nitritos con aminas provenientes de la carne (11). Algunas nitrosaminas son reconocidamente tóxicas para la célula B (12). Estudios revelan que el consumo de alimentos con alto contenido en nitritos y nitrosaminas se asocia positivamente con el riesgo de DM 1 en diversas poblaciones (13-15).

En otro estudio de cohorte prospectivo, que incluyó 13.100 mujeres, sin antecedentes de enfermedad cardiovascular, cáncer, DM 2 o historia de diabetes gestacional; se observó que un patrón alimentario prudente (caracterizado por alto consumo de frutas, vegetales verdes y carnes blancas) se asoció significativa e inversamente con el riesgo de diabetes gestacional. En contraste, un patrón alimentario occidental (caracterizado por alta ingesta de carnes rojas, carnes procesadas, hidratos de carbono refinados, dulces, frituras y pizza) se asoció significativa y positivamente con la diabetes gestacional. Esta asociación se mantuvo después de ajustar por edad, paridad, IMC previo a embarazo y otras covariables (16).

Un estudio prospectivo realizado en mujeres diabéticas tipo 2, desde 1980 al 2000 (54.455 personas-año de seguimiento) evidenció una asociación significativa entre la ingesta de hierro hem y carnes rojas con mayor riesgo de enfermedad coronaria fatal, revascularización coronaria y cardiopatía coronaria total. Esta observación fue independiente de otros factores de riesgo convencionales para cardiopatía coronaria (17).

\section{Hipertensión arterial y enfermedad cardiovascular}

La HTA es una de las principales causas de cardiopatía coronaria, accidente vascular encefálico (AVE) y mortalidad temprana. Existen algunas poblaciones en el mundo donde la presión arterial no se eleva con la edad y donde la alta prevalencia de HTA observada en otros países occidentales no ha sido evidenciada. Sin embargo cuando estas poblaciones migran a sectores urbanos, su presión arterial se eleva, indicando que la HTA es un problema de origen ambiental (18). La dieta juega un importante rol en la modulación de la presión arterial en personas hipertensas y normotensas $(19,20)$.

El estudio de alimentación DASH (Dietary Approaches to Stop Hypertension) encontró que una combinación de dieta rica en frutas, vegetales, y productos lácteos bajos en grasas totales y saturadas puede disminuir sustancialmente los niveles de presión arterial sistólica y diastólica en 459 hombres y mujeres, blancos y negros moderadamente hipertensos. Estudios han mostrado que vegetarianos tienen niveles más bajos de presión arterial que los no vegetarianos y la adición de carne a la dieta vegetariana aumenta la presión arterial $(18,19)$.

El estudio CARDIA (Coronaty Artery Risk Development in Young Adults), es un estudio poblacional, multicéntrico, prospectivo de la evolución de los factores de riesgo cardiovascular en negros y blancos, hombres y mujeres ( $n=4.304$ entre 18 y 30 años), con un seguimiento durante 15 años se encontró que la incidencia de HTA varió desde un $12 \%$ en mujeres blancas hasta un $33 \%$ en hombres negros. La ingesta de alimentos de origen vegetal estuvo inversamente relacionada con HTA luego de ajustar por edad, sexo, raza, ingesta de energía, factores de riesgo cardiovascular y otros factores potencialmente confundentes (20).

El consumo de carne se relacionó positivamente con el riesgo de desarrollar HTA, incluso después de ajustar por otro posibles factores. El consumo de carnes rojas procesadas se asoció con un mayor riesgo (20-40\%) de desarrollar HTA, en comparación con el consumo de carnes rojas sin procesar. En este estudio el consumo de pescado no se relacionó con HTA, sin embargo estudios previos han reportado una relación inversa entre ingesta de pescado y AVE (21). Dado el limitado consumo de pescado, el subgrupo que consumió pescado, incluyó 
pescado fresco y procesado. Es importante destacar que la composición del pescado procesado puede ser similar al de la carne, rico en grasas saturadas (19).

\section{Composición de ácidos grasos en la dieta}

La relación n-6/n-3PUFA alta también se considera un marcador de riesgo elevado de enfermedad cardiovascular (22-24). Un metaanálisis de cuatro cohortes de estudio prospectivo $(n=140.000)$ mostró que un aumento de un $2 \%$ en la ingesta energética proveniente de ácidos grasos trans se asocia con un aumento de un $23 \%$ en la incidencia de enfermedad cardiovascular (6).

La importancia en la dieta de PUFA n-3 se resaltó en estudios de esquimales, quienes consumen una gran cantidad de animales marinos, contribuyendo a un alto consumo, que parece ser responsable de la baja incidencia de enfermedad cardiovascular en esta población con un alto consumo de grasas (22-26). El beneficio de PUFA n-3 en la enfermedad cardiovascular ha sido demostrado en animales y en humanos (25).

Un estudio realizado en Australia en 147 hombres sanos no fumadores entre 20 y 55 años, quienes fueron clasificados en cuatro sub grupos: (HME) consumidores de $285 \mathrm{~g} /$ día o más de carne; (MME) consumidores de $<260$ g/día de carne; ovo-lacto vegetarianos quienes no consumen carne, pero si consumen huevos y productos lácteos; mientras que los veganos no consumen carne, huevos ni productos lácteos. El grupo HME consumía significativamente más ácidos grasos saturados, el grupo vegano consumió significativamente menos ácidos grasos saturados y grasas totales. Los niveles plasmáticos de ácidos grasos saturados fueron significativamente menores en el grupo vegano. Los nhveles de PUFA total fue mayor en el grupo vegano. En el grupo HME y MME hubo mayores niveles plasmáticos de ácido araquidónico en comparación con el grupo ovolacto vegetariano (22).

Existe evidencia que los estrógenos reducen la susceptibilidad de las LDL a la oxidación. Los productos de soya como el tofu, contienen fitoquímicos con propiedades similares a los estrógenos (llamados fitoestrógenos). Las isoflavonoides genistein y daidzein se forman en el cuerpo por la de conjugación de los glucósidos presentes en el poroto de soya $(27,28)$, previniendo el riesgo cardiovascular.

Un estudio realizado en Australia de ensayo clínico randomizado incluyó a 45 hombres sanos entre 35 y 62 años. Recibieron dos tipos de dieta similar en energía, proteínas, grasas, hidratos de carbono, alcohol y fibra, con la única diferencia en la fuente de proteínas animal y vegetal. Los resultados mostraron que el colesterol total y los triglicéridos plasmáticos fueron significativamente menores y la fase de oxidación de LDL in vitro fue significativamente más larga en la dieta con tofu comparado con la dieta con carne (27).

\section{Factores de riesgo trombótico}

La trombosis arterial juega un rol importante en la cardiopatía coronaria manifestado como angina inestable, infarto agudo al miocardio (IAM) y muerte súbita. Los factores hemostáticos, factor VII y fibrinógeno, son importantes en este proceso (27-29). La concentración de fibrinógeno es también un determinante mayor de la viscosidad plasmática. Estudios cruzados han mostrado relación entre la cardiopatía coronaria y los niveles plasmáticos del factor VII y fibrinógeno (27).

Los estudios en esta área han mostrado resultados dispares. Un estudio australiano descrito previamente que comparó el consumo de tofu versus el consumo de carne, los factores hemostáticos, factor VII y fibrinógeno, y lipoproteína (a) no se afectaron significativamente por la dieta con tofu (27).

La carne es la mayor fuente dietaría de ácido araquidónico (AA). Estudios en vegetarianos y omnívoros también han reportado pequeñas diferencias en los niveles de AA plasmáticos o plaquetarios (omnívoros con un 5-10\% más en la proporción de AA) (29).

Otro estudio australiano que incluyó 139 hombres sanos (veganos $n=18$, ovolacto vegetarianos $n=43$, MME n=60, HME n= 18), entre 20 y 55 años, comparó a los consumidores de carne habituales y los vegetarianos habituales, y evidenció que los consumidores de carne tuvieron factores de riesgo cardiovascular significativamente mayores, comparado con el grupo vegetariano, incluyendo un aumento del IMC, relación cintura-cadera, colesterol plasmático total, low density lipoprotein (LDL), triglicéridos, relación colesterol total / High density lipoprotein (HDL), y actividad del factor VII plasmático. El grupo vegano tuvo un recuento plaquetario significativamente menor comparado con el grupo ovolacto vegetariano. Los veganos tuvieron una actividad del factor VII plasmático significativamente menor comparado con MME (29).

\section{Marcadores de inflamación y disfunción endotelial}

En pacientes con enfermedad coronaria, los niveles de ácidos grasos trans en las membranas (biomarcador de ingesta de ácidos grasos trans) han sido independientemente asociados con la activación de la respuesta inflamatoria sistémica, incluyendo un aumento sustancial en los niveles de interleukina-6, TNF-a, receptores de TNF y proteínas que atraen monocitos. Concordantemente, diversos estudios sugieren que los ácidos grasos trans 
causan disfunción en el endotelio, con aumento en los niveles de marcadores de disfunción endotelial (moléculas de adhesión intracelular y vascular, E-selectina) y con un deterioro de la función endotelial reflejado en una disminución del flujo mediado por vasodilatación en la arteria radial (6).

La dieta juega un importante rol en el desarrollo de alteraciones metabólicas e inflamatorias. Diversos estudios han examinado el efecto de la soya en los niveles plasmáticos de mediadores inflamatorios y de función endotelial. La soya contiene fibra, PUFA y fitoestrógenos, cada uno asociado con bajos niveles de marcadores inflamatorios y mejoría en la función endotelial (30). Las isoflavonas estimulan la actividad de la óxido nítrico (NO) sintetasa, induciendo vasodilatación vía NO. Isoflavonas también tienen un efecto antitrombótico y antiaterogénico. Por ejemplo, genistein y daidzein disminuyen la proteína 1 que atrae los monocitos y la agregación plaquetaria inducida por colágeno (28).

Un estudio randomizado y cruzado incluyó a 42 mujeres post menopáusicas con síndrome metabólico. Las participantes fueron asignadas las que consumían una dieta control, una dieta con proteína de soya, una dieta con poroto de soya, cada una por ocho semanas. Los resultados mostraron que el consumo de poroto de soya reduce las concentraciones plasmáticas de E-selectina, IL-18, TNF-a y PCR e incrementa los niveles plasmáticos de óxido nítrico en mujeres post menopáusicas con síndrome metabólico (30).

Otros estudios han demostrado en mujeres obesas una mejoría en la función endotelial asociada a una disminución en el peso (31). Una publicación reciente comparó el efecto de la proteína de soya, proveniente de diferentes productos procesados con el riesgo cardiovascular y función endotelial en sujetos hipercolesterolémicos, no mostrando diferencias significativas en la presión arterial, función endotelial, colesterol total, very low density lipoprotein (VLDL), triglicéridos, Apo B, proteína $\mathrm{C}$ reactiva (PCR) (32).

La disparidad encontrada en los resultados de los estudios con soya puede explicarse por la gran variabilidad en el contenido de isoflavonas de la soya. Entre los factores que afectan la concentración de isoflavonas se encuentran la variedad de soya, lugar geográfico, condiciones ambientales, procesamiento industrial (32).

Una revisión reciente destaco el beneficio del consumo de nueces en la salud cardiovascular, este alimento es fuente alta en energía, grasa total y ácidos grasos insaturados. Estudios de ensayo clínicos y de corte trasversal han mostrado en grupos con alto consumo de esta oleaginosa bajos niveles de concentración en circulación de moléculas inflamatorias (citoquinas y PCR) y altos niveles de adiponectina un potente antiinflamatorio. Además de mejoría en la función endotelial (33).

\section{Mortalidad}

Un estudio realizado en USA, Reino Unido y Alemania, con el objetivo de comparar mortalidad en vegetarianos y no vegetarianos, incluyó 76.172 hombres y mujeres entre 16 y 89 años al momento de ser reclutados. Se consideraron vegetarianos quienes no consumían carne ni pescado ( $\mathrm{n}=27.808)$. Después de 10.6 años de seguimiento se observó que los vegetarianos tuvieron $24 \%$ menos mortalidad por cardiopatía coronaria en comparación con los no vegetarianos. No hubo diferencias significativas entre vegetarianos y no vegetarianos en la mortalidad por otras causas.

El estudio EPIC-Postdam (European Prospective Investigation into Cancer and Nutrition-Postdam) cohort, $(n=26.238)$ es parte de un largo estudio de cohorte, prospectivo, europeo (EPIC), encontró que un patrón alimentario con un alto consumo de hidratos de carbono no refinados, frutas frescas, jugo de frutas, cereales y vegetales combinado con un bajo consumo de carnes procesadas, mantequilla, queso rico en grasas, margarina y carnes rojas, muestra una asociación inversa con mortalidad cardiovascular (21).

Otro estudio prospectivo de seguimiento por 15 años de 6000 sujetos daneses, con un patrón de alimentación prudente, definido por consumo frecuente de pan integral, vegetales, fruta y pescado evidenció una menor mortalidad por todas las causas y menor riesgo cardiovascular (34).

\section{DISCUSIÓN}

Existe una amplia evidencia científica que respalda el rol primario de la dieta en la prevención de las enfermedades cardiovasculares. Los protocolos nutricionales fomentan como recomendaciones cardio-protectoras el incremento en la ingesta de fruta y verduras ( 9 a 11 porciones por día), fibra dietaría ( $25 \mathrm{~g}$ /día), consumo regular de ácidos grasos poliinsaturados omega 3 en pescados de aguas frías (al menos 2 veces por semana), plantas con esteroles/estanoles ( $2 \mathrm{~g} /$ día), nueces ( 1 onza/día) y sustituir la proteína animal por proteína de soya para reducir la ingesta de grasa saturada (35). Recomendaciones nutricionales caracterizadas por un alto consumo de alimentos de origen vegetal y moderado consumo de origen animal.

Entre los principales factores que aumentan el riesgo de morbi-mortalidad cardiovascular están la DM 2 y la HTA. La evidencia muestra que el consumo de ácidos grasos saturados provenientes de alimentos de 
origen animal especialmente las carnes rojas y lácteos está asociado con un aumento en la prevalencia de diabetes. Se postula que la insulino sensibilidad depende de la composición de ácidos grasos en las membranas lipídicas, la grasa insaturada la aumenta y la grasa trans y saturada la disminuye (4). Varios estudios relacionan el consumo de carnes procesadas con el aumento de DM $2(5,9)$, las nitrosamidas presentes en este tipo de productos alimentarios han sido relacionadas con este efecto por ser potencialmente tóxicas para las células beta del páncreas.

La dieta DASH recomendada para el manejo de la hipertensión se caracteriza por ser rica en frutas, vegetales, y baja en alimentos con alto contenido de grasas saturadas. La disminución sustantiva de los niveles de presión arterial sistólica y diastólica que se presentan con estas recomendaciones se asocia fuertemente con el alto consumo de alimentos de origen vegetal. Varios estudios comparativos entre grupos vegetarianos y no vegetarianos reafirman estos resultados $(18,19)$.

La relación entre los ácidos grasos de la dieta y el riesgo cardiovascular es de los factores más estudiados. La ingesta de grandes cantidades de grasas saturadas y ácidos grasos trans junto con bajos niveles de PUFA particularmente de cadena larga omega -3 , tiene un gran impacto en la enfermedad cardiovascular. El alto consumo de ácidos grasos saturados y trans característico de los alimentos de origen animal, elevan los niveles de LDL, reducen los niveles de HDL, aumenta la relación colesterol total/HDL, aumentan los niveles sanguíneos de triglicéridos, lipoproteína A y reduce el tamaño de las partículas de $\operatorname{LDL}(6,22)$.

El alto consumo de ácidos grasos insaturados característico de los alimentos de origen vegetal disminuyen el riesgo cardiovascular, entre los mecanismos reportados incluyen: una disminución de la presión arterial; disminución de triglicéridos plasmáticos; disminución de trombo génesis; efectos antiinflamatorios; efectos antiarrítmicos; relajación de los vasos sanguíneos y disminución de la insulino resistencia $(25,26)$.

Los factores de riego trombótico como el factor VII, el fibrinógeno y la producción de de tromboxano A2 (TXA2), han sido relacionados con el tipo de dieta y el riesgo cardiovascular. Estudios muestran efectos favorables en grupos vegetarianos y efectos desfavorables en grupos no vegetarianos. La agregación plaquetaria es un evento temprano en el desarrollo de la trombosis. Se inicia con la producción de TXA2 producido desde ácido araquidónico (AA), (20:4n-6), un PUFA n-6 de cadena larga, que se encuentra principalmente en la carne (29).

Se requiere mayor número de estudios que rela- cionen el consumo de alimentos con los marcadores de inflamación y disfunción endotelial. Se ha encontrado un afecto desfavorecedor de las grasas trans (6), discutido de la soya (30-32) y favorecedor de las nueces (33).

Finalmente, los resultados hasta el momento concuerdan en encontrar una menor mortalidad cardiovascular en grupos con alto consumo de alimentos de origen vegetal comparado con aquellos de alto consumo de alimentos de origen animal $(21,34)$.

\section{CONCLUSIÓN}

La muerte por enfermedades cardiovasculares sigue aumentando de manera alarmante en el mundo, varias de sus causas como los hábitos de alimentación inadecuados, la baja actividad física y el consumo de tabaco son prevenibles, los resultados de esta revisión reafirman la importancia de emitir el mensaje a nivel poblacional de recomendar una disminución en el consumo de carnes rojas, principalmente procesadas, disminuir el consumo de grasas saturadas, trans, así como estimular el consumo de cereales integrales, frutas, vegetales y pescado para generar un gran impacto en una disminución de la morbi-mortalidad global y cardiovascular.

\section{RESUMEN}

El aumento de la mortalidad cardiovascular en el último tiempo está fuertemente asociado a cambios en los hábitos de alimentación. La dieta occidental caracterizada por una alta ingesta de alimentos de origen animal, presenta mayor riesgo de diabetes tipo 2 (DM 2), hipertensión arterial (HTA), aumento de marcadores de disfunción endotelial y factores protrombóticos. La búsqueda se realizó en la biblioteca online Pubmed y en referencias de otras revisiones. Los criterios de inclusión fueron: artículos de 1999 en adelante, ensayos clínicos aleatorizados-controlados, estudios caso-control, revisiones sistemáticas y meta análisis. En grupos con alto consumo de alimentos de origen vegetal se ha evidenciado una menor morbi-mortalidad cardiovascular, comparado con los grupos con alta ingesta de alimentos de origen animal. La evidencia apoya que la disminución en el consumo de carnes rojas, grasas saturadas y grasas trans, así como el aumento en el consumo de cereales integrales, frutas, vegetales y pescado, disminuye la morbi-mortalidad global y cardiovascular.

Palabras clave: alimentos de origen animal; alimentos de origen vegetal; HTA, DM2; mortalidad cardiovascular.

Dirigir la correspondencia a:

Srta.

Ángela Pino Labrador 
Miguel Claro 967 Depto. 304

Providencia

Santiago - Chile

Fonos: 07-8998306 - 3256318

E-mail: apino@inta.cl

\section{BIBLIOGRAFÍA}

1. Mann N. Dietary lean red meat and human evolucion. Eur J Nutr 2000; 39: 71-79.

2. Key TJ, Fraser GE, Thorogood M, et al. Mortality in vegetarians and non vegetarians: a collaborative analysis of 8300 deaths among 76000 men and women in five prospective studies. Pub Health Nutr 1998; 1: 33-41.

3. Feskens EJ, van Dam RM: Dietary fat and the etiology of type 2 diabetes: an epidemiological perspective. Nutr Metab Cardiovasc Dis 1999; 9:87-95,

4. Storlien LH, Baur LA, Kriketos AD, Pan DA, Clooney GJ, Jenkins AB, Calvert GD, Campbell LV: Dietary fats and insulin action. Diabetol 1996; 39:621-631.

5. Van dam RM, Willet WC, Rimm EB, et al. Dietary Fat and Meat Intake in Relacion to Risk of Type 2 Diabetes in Men. Diabetes Care 2002; 25: 417 424.

6. Mosaffarian D, Katan MB, Ascherio A, et al. Trans Fatty Acids and Cardiovascular Disease. N Engl J Med 2006; 354: 1601-13.

7. Zhang C, Schulze MB, Solomon CG, et al. Diabetologia 2006; 49: 2604-2613.

8. Nogi A, Yang J, Li L, et al. Plasma n-3 Polyunsaturated Fatty Acid and Cardiovascular Disease Risk Factors in Japanese, Korean and Mongolian Workers. J Occup Health 2007; 49: 205-216.

9. Snowdon DA, Phillips RL: Does a vegetarian diet reduce the occurrence of diabetes? Am J Public Health 1985; 75:507-512,

10. Knight TM, Forman D, Al-Dabbagh SA, Doll $\mathrm{R}$ : Estimation of dietary intake of nitrate and nitrite in Great Britain. Food Chem Toxicol 1987; 25:277-285,

11. Lijinsky W: N-Nitroso compounds in the diet. Mutat Res 1999; 443:129-138,

12. LeDoux SP, Hall CR, Forbes PM, Patton NJ, Wilson GL: Mechanisms of nicotinamide and thymidine protection from alloxan and streptozocin toxicity. Diabetes 1988; 37:1015-1019,

13. Dahlquist GG, Blom LG, Persson LA, Sandstrom AI, Wall SG: Dietary factors and the risk of developing insulin dependent diabetes in childhood. BMJ 1990; 300:1302-1306,

14. Virtanen SM, Jaakkola L, Ra“sa”nen L, Ylo“nen K,
Aro A, Lounamaa R, Åkerblom HK, Tuomilehto $\mathrm{J}$ : Nitrate and nitrite intake and the risk for type 1 diabetes in Finnish children: Childhood Diabetes in Finland Study Group. Diabet Med 1994 11:656-662,

15. Helgason T, Jonasson MR: Evidence for a food additive as a cause of ketosis-prone diabetes. Lancet 1981; 2:716-720.

16. Trevisan M, Krogh V, Freudenheim J, Blake A, Muti P, Panico S, Farinaro E, Mancini M, Menotti A, Ricci G: Consumption of olive oil, butter, and vegetable oils and coronary heart disease risk factors: the Research Group ATS-RF2 of the Italian National Research Council. JAMA 263:688-692, 1990

17. Lu Qi, Van dam RM, Rexrode K, et al. Heme Iron From Diet as a Risk Factor for Coronary Heart Disease in Women With Type 2 Diabetes. Diabetes Care 2007; 30: 101-106.

18. Elliot P. Protein intake and blood pressure in cardiovascular disease. Proc Nutr Soc 2003; 62: 495-504.

19. Steffen LM, Kroenke CH, Yu X, et al. Associations of plant food, dairy product, and meat intakes with 15 -y incidence of elevated blood pressure in young black and white aduts: the Coronary Artery Risk Development in Young Adults (CARDIA) Study. Am J Clin Nutr 2005; 82: 1169-77.

20. Famodu AD, Osilesi O, Makinde YO, et al. Blood Pressure and Blood Lipid Levels Among Vegetarian, Semi-Vegetarian, and Non-Vegetarian Native Africans. Clin Biochem 1998; 31: 545-549.

21. Drogan D, Hoffmann K, Schulz M, et al. A Food Pattern Predicting Prospective Weight Change Is Associated with Risk of fatal but Not with Nonfatal Cardiovascular Disease. J Nutr 2007; 137: 1961-1967.

22. Mann N, Pirotta Y, O’Connell S, et al. Fatty Acid Composition of Habitual Omnivore and Vegetarian Diets. Lipids 2006; 41: 637-646.

23. Hu FB, Manson JE, Willet WC. Types oj Dietary Fat and Risk of Coronary Heart Disease: A Critical Review. J Am College Nutr 2001; 20, N 1: 5-19.

24. Hu FB, Stampfer MJ, Manson JE, et al. Dietary saturated fats and their food sources in relation to the risk of coronary heart disease in women. Am J Clin Nutr 1999; 70: 1001-1008.

25. Nogi A, Yang J, Li L, et al. Plasma n-3 Polyunsaturated Fatty Acid and Cardiovascular Disease Risk Factors in Japanese, Korean and Mongolian Workers. J Occup Health 2007; 49: 205-216.

26. Kris-Etherton PM, Harris WS, Lawrence J. Fish Consumption, Fish Oil, Omega-3 Fatty Acids, and 
Cardiovascular Disease. Arterioscler Thromb Vasc Biol 2003;23: e20-e31.

27. Ashton E, Dalais FS, Ball MJ. Effect of Meat Replacement by Tofu on CHD Risk Factors Including Copper Induced LDL Oxidation. J Am College Nutr 2000; 19, N6: 761-767.

28. Tempfer CB, Bentz EK, Leodolter S, et al. Phytoestrogens in clinical practice: a reciew of the literature. Fertility and Sterility 2007;87(6):1243-1249.

29. Li D, Sinclair A, Mann N, et al. The association of diet and Thrombotic risk factors in healthy male vegetarians and meat-eaters. Eur J Clin Nutr 1999;53:612-619.

30. Azadrakht L, Kimiagar M, Mehrabi Y, et al. Soy Consumption, Markers of inflammation, and Endothelial Function. Diabetes Care 2007;30:967973.

31. Yamashita T, Sasahara T, Pomeroy SE, et al. Arterial Compliance, Blood Pressure, Plasma leptin, and Plasma lipids in Women Are Improved With Weight Reduction Equally With a meat-Based Diet and a Plant-Based Diet. Metabolism 1998; 47, (11): 1308-1314.

32. Matthan NR, Jalbert SM, Ausman LM. Effect of soy protein from differently processed products on cardiovascular disease risk factors and vascular endothelial function in hypercholesterolemic subjects. Am J Clin Nutr 2007; 85: 960-966.

33. Ros E. Nut's and novel biomarkers of cardiovascular disease. Am J Clin Nutr 2009 May; 89 (5):1649S56S. Epub 2009 Mar 25.

34. Osler M, Heitmann BL, Gerdes LU, et al. Dietary patterns and mortality in Danish men and women: a prospective observational study. Br J Nutr 2001; 85: 219-225.

35. Retelny VS, Neuendorf A, Roth JL. Nutrition protocols for the prevention of cardiovascular disease. Nutr Clin Pract 2008; 23(5):468-76. 